\title{
Electron Trajectories in Molecular Orbitals
}

\author{
Isaiah Sumner ${ }^{\mathrm{a}}$ and Hannah Anthony ${ }^{\mathrm{a}}$ \\ ${ }^{a}$ Department of Chemistry and Biochemistry, James Madison University, Harrisonburg, VA 22807, \\ USA \\ Correspondence: \\ I. Sumner \\ 901 Carrier Drive, MSC 4501 \\ Harrisonburg, VA 22807 \\ Phone: +1-540-568-6670 \\ Email: sumneric@jmu.edu
}

\begin{abstract}
The time-dependent Schrödinger equation can be rewritten so that its interpretation is no longer probabilistic. Two well-known and related reformulations are Bohmian mechanics and quantum hydrodynamics. In these formulations, quantum particles follow real, deterministic trajectories influenced by a quantum force. Generally, trajectory methods are not applied to electronic structure calculations, since they predict that the electrons in a ground state, real, molecular wavefunction are motionless. However, a spin-dependent momentum can be recovered from the non-relativistic limit of the Dirac equation. Therefore, we developed new, spin-dependent equations of motion for the quantum hydrodynamics of electrons in molecular orbitals. The equations are based on a Lagrange multiplier, which constrains each electron to an isosurface of its molecular orbital, as required by the spin-dependent momentum. Both the momentum and the Lagrange multiplier provide a unique perspective on the properties of electrons in molecules.
\end{abstract}

Keywords: Bohmian mechanics, quantum hydrodynamics, molecular orbitals, Dirac equation, electronic structure

\section{Introduction}

Bohmian mechanics and quantum hydrodynamics[1]-[6] are trajectory based formulations of quantum mechanics[7], wherein quantum particles follow real, "classical-like" paths. In Bohmian mechanics, a quantum particle moves along a real trajectory and is influenced by a nonlocal "quantum-force," which is responsible for all quantum behavior (tunneling, zero-point energy, etc.). In quantum hydrodynamics (QHD), the wavefunction is reinterpreted as a probability fluid composed of particles that also follow real trajectories. Both one-particle QHD and Bohmian mechanics are formally equivalent to the time-dependent Schrödinger equation (TDSE) and therefore make identical predictions to the TDSE. However, they are useful as computational and interpretational tools and have subsequently been developed into a diverse set of methods. Some of these couple quantum and classical systems[8]-[13], construct wavefunctions [6], [14], and simulate reactive scattering[15]-[21], among other applications [22]-[30]. Bohm's formalism has even seen a recent revival in experimental fluid mechanics. A set of experiments have shown how droplets on fluid surface act as classical analogs of Bohmian particles [31]-[37], although the analogy is imperfect [38]. Despite their popularity in other areas of chemical physics, trajectory methods have been largely (but not completely[39]-[44]) ignored in electronic structure theory. A likely reason for this lack of interest is that a goal of electronic structure theory is to solve for the ground state, electronic eigenfunction of a molecular Hamiltonian. Traditional Bohmian 
mechanics and QHD predict that particles in real eigenfunctions of Hamiltonians (i.e. static stationary states) are motionless and it is difficult to interpret motionless electrons. However, it is possible to reformulate Bohmian mechanics and QHD to allow for motion even in static stationary states. One route is to allow the particles to move in the complex plane[45]-[55][56]. Another route is to include particle spin [57]-[60]. The second route has the advantage that the particle trajectories remain fully real. Thus, we have further developed the spin-dependent methods into a new technique suitable to describe electron trajectories in molecular orbitals.

\section{Discussion}

$\underline{\text { Formalism }}$

The first step in deriving the QHD and Bohmian equations of motion[5], [6] is to write the wavefunction in its polar form

$$
\Psi(\boldsymbol{r}, t)=A(\boldsymbol{r}, t) e^{i S(\boldsymbol{r}, t) / \hbar}
$$

where $\mathrm{A}(\mathbf{r}, \mathrm{t})^{2}=\rho(\mathbf{r}, t)$ - the probability density, and $\mathrm{A}(\mathbf{r}, \mathrm{t})$ and $S(\mathbf{r}, t)$ are purely real functions. The wavefunction is then plugged into the time-dependent Schrödinger equation (TDSE),

$$
i \hbar \frac{\partial}{\partial t} \Psi(\boldsymbol{r}, t)=\widehat{H} \Psi(\boldsymbol{r}, t),
$$

and the real and imaginary parts are separated, resulting in two equations:

$$
\begin{gathered}
-\frac{\partial S}{\partial t}=\frac{(\nabla S)^{2}}{2 m}+V-\frac{\hbar^{2}}{2 m} \frac{\nabla^{2} A}{A} \\
\frac{\partial \rho}{\partial t}=-\nabla \cdot\left(\rho \frac{\nabla S}{m}\right)=-\nabla \cdot \mathcal{J} .
\end{gathered}
$$

( $\mathbf{r}$ and $t$ will be omitted for the remainder of this derivation.) Equation (4) is the probability continuity equation and $\mathcal{J}=\frac{-i \hbar}{2 m}\left(\Psi^{*} \nabla \Psi-\Psi \nabla \Psi^{*}\right)-$ the probability flux. As written, $\mathcal{J}$ contains a gauge symmetry [61], [62] because adding a curl term to $\mathcal{J}$ (i.e., $\tilde{\mathcal{J}}=\mathcal{J}+\nabla \times \mathcal{F}$ ) will not affect Eq. (4); the divergence of a curl is identically zero.

In hydrodynamics, the continuity equation describes the flow of an incompressible fluid, where $\mathcal{J}=\rho \mathbf{v}, \rho$ is the fluid density, and $\mathbf{v}$ is the flow velocity. In QHD, $\rho$ is the density of the "probability fluid" and $\nabla S$ is the momentum of particles within that fluid. This ansatz also shows that Eq. (3) is a Hamilton-Jacobi equation and $-\frac{\partial S}{\partial t}$ is the total (kinetic plus potential) energy. However, Eq. (3) contains a non-classical term, $-\frac{\hbar^{2}}{2 m} \frac{\nabla^{2} A}{A}=-\frac{\hbar^{2}}{2 m} \frac{\nabla^{2} \sqrt{\rho}}{\sqrt{\rho}}$. This term is the "quantum 
potential" and is responsible for all quantum effects (zero-point energy, tunneling, etc.). Finally, since $\frac{d}{d t}=\frac{\partial}{\partial t}+\mathbf{v} \cdot \nabla$, the gradient of Eq. (3) is

$$
-\nabla\left(V-\frac{\hbar^{2}}{2 m} \frac{\nabla^{2} \sqrt{\rho}}{\sqrt{\rho}}\right)=F_{C l}+F_{Q}=m a,
$$

a Newtonian-like equation of motion where $\mathbf{a}$ is acceleration and $F_{C l}$ and $F_{Q}$ are classical and quantum forces. These equations allow quantum mechanics to be reinterpreted as a particle based theory (i.e., no wavefunction), where the particles are influenced by a non-local potential[3]-[5].

Equations (3), (4) and (5) give counterintuitive results when the wavefunction is a static stationary state. In this case, the wavefunction is

$$
\Psi=\phi_{n}(\boldsymbol{r}) e^{-i E_{n} t / \hbar}
$$

where $E_{n}$ is the nth energy eigenvalue and $\phi_{n}$ is the nth energy eigenstate. By comparing Eq. (6) to Eq. (1), it is clear that $S=-E t$ and $\nabla S=\mathbf{p}=0$. Thus, a particle in a real energy eigenstate is motionless. This feature disappears when spin is accounted for[57].

Spin-Dependent Quantum Hydrodynamics

Spin-dependent QHD (SD-QHD) [57]-[60] is based on the Dirac Equation, the fundamental equation for a spin-1/2 particle like an electron[63]:

$$
i \hbar \frac{\partial}{\partial t}\left(\begin{array}{l}
\psi \\
\chi
\end{array}\right)=-i \hbar c \boldsymbol{\sigma} \cdot \nabla\left(\begin{array}{l}
\chi \\
\psi
\end{array}\right)+V\left(\begin{array}{l}
\psi \\
\chi
\end{array}\right)+m c^{2}\left(\begin{array}{c}
\psi \\
-\chi
\end{array}\right)
$$

where, $\boldsymbol{\sigma}=\sigma_{x} \hat{\boldsymbol{\imath}}+\sigma_{y} \hat{\boldsymbol{\jmath}}+\sigma_{z} \widehat{\boldsymbol{k}}$ are the $2 \times 2$ Pauli spin matrices; $\hat{\boldsymbol{\imath}}, \hat{\boldsymbol{\jmath}}$, and $\widehat{\boldsymbol{k}}$ are the unit vectors in the $\mathrm{x}, \mathrm{y}$ and $\mathrm{z}$-directions; $\mathrm{c}$ is the speed of light; and $\psi$ and $\chi$ are two-component spinors. (Equation (7) is written in the absence of an external electric field.) The Dirac equation also obeys a probability continuity equation[63]:

$$
\frac{\partial}{\partial t} P=-\nabla \cdot c\left(\psi^{\dagger} \boldsymbol{\sigma} \chi+\chi^{\dagger} \boldsymbol{\sigma} \psi\right)=-\nabla \cdot \mathcal{J}_{D}
$$

where $P=\left(\begin{array}{ll}\psi^{\dagger} & \chi^{\dagger}\end{array}\right)\left(\begin{array}{l}\psi \\ \chi\end{array}\right)$. In the non-relativistic limit[63],

$$
\chi \simeq \frac{-i \hbar \sigma \cdot \nabla}{2 m c} \psi
$$

and the expression for $\mathcal{J}_{D}$ becomes 


$$
\mathcal{J}_{D}=\frac{-i \hbar}{2 m}\left(\psi^{\dagger} \nabla \psi-\left(\nabla \psi^{\dagger}\right) \psi\right)+\frac{\hbar}{2 m} \nabla \times\left(\psi^{\dagger} \boldsymbol{\sigma} \psi\right)
$$

If $\psi$ is an eigenstate of $\sigma_{z}$ (i.e., a spin-up or spin-down electron), Eq. (10) reduces to[57], [61], [62]

$$
\mathcal{J}_{D}=\frac{-i \hbar}{2 m}\left(\Psi^{*} \nabla \Psi-\Psi \nabla \Psi^{*}\right) \pm \frac{\hbar}{2 m} \nabla \times(\rho \widehat{\boldsymbol{k}})=\mathcal{J} \pm \frac{\hbar}{2 m} \nabla \times(\rho \widehat{\boldsymbol{k}})
$$

which fixes the gauge symmetry of $\mathcal{J}$ [61], [62]. Finally, using the hydrodynamic analogy, Eq. (11) can be rewritten as [57]

$$
\mathcal{J}_{D}=\rho \boldsymbol{v}=\rho\left(\frac{\nabla S}{m} \pm \frac{\hbar}{2 m} \frac{\nabla \times(\rho \widehat{\boldsymbol{k}})}{\rho}\right)=\rho\left(\frac{\nabla S}{m}+\frac{1}{m} \nabla \log \rho \times \boldsymbol{s}\right)
$$

where $\mathbf{s}= \pm \frac{\hbar}{2} \widehat{\boldsymbol{k}}$ is the spin vector in the z-direction. Therefore, the particle's momentum becomes $\mathbf{p}=\nabla S+\nabla \log \rho \times \mathbf{s}$, which is non-zero even in a static stationary state, in which case

$$
\mathbf{p}=\nabla \log \rho \times \mathbf{s}
$$

Geometric considerations show that the spin-dependent momentum is always perpendicular to the spin vector, $\mathbf{s}$, and parallel to an isosurface of the probability density, $\rho$ ( $\nabla \log \rho$ is perpendicular to the isosurfaces of $\rho$ ). Thus, an electron in a static stationary state travels along a level curve (contour) of $\rho$, and spin-up/spin-down $(\alpha$ and $\beta$ ) electrons orbit in opposite directions. Additionally, $\mathbf{p}$ approaches infinity as the electron approaches wavefunction nodes. Since the direction of $\nabla \log \rho$ flips on either side of a node ( $\nabla \log \rho$ points towards increasing $\rho$ ), SD-QHD trajectories cannot cross nodes. They share this property with standard Bohmian mechanics.

\section{One-electron atoms: $\mathrm{H}$}

The SD-QHD has been extensively applied to the hydrogen atom in Refs. [57]-[59]. One of the main results is reproduced here. An electron in a $1 s$-orbital has the following time-dependent wavefunction:

$$
\Psi(\boldsymbol{r}, t)=\frac{1}{\sqrt{\pi a_{0}^{3}}} e^{-r / a_{0}} e^{-i E t}=A(\boldsymbol{r}) e^{-i E t}
$$




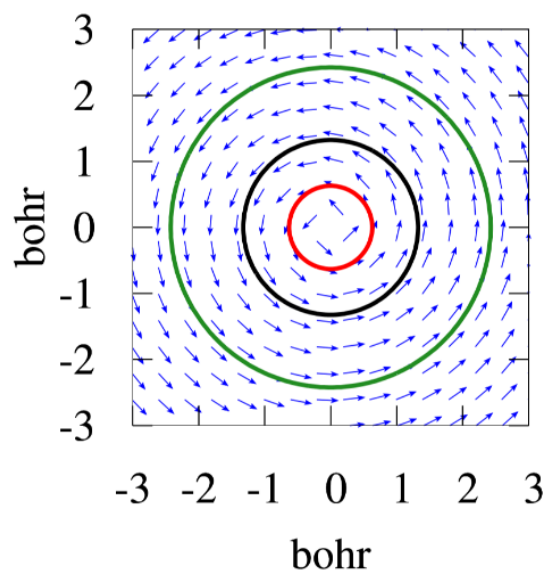

Figure 1 Level curves of the $1 \mathrm{~s}$ orbital density in the $z=0$ plane. The nucleus is at the origin and distances are in atomic units. The arrows represent the electron's momentum vector field, $\nabla \log \rho \times \boldsymbol{s}$, and have been scaled for clarity. The electron travels exclusively along level curves of $\rho$, which are circles centered around the nucleus.

where $a_{0}$ is the Bohr radius. The electron's momentum in spherical coordinates is:

$$
\boldsymbol{p}=\nabla \log \rho \times \boldsymbol{s}=\frac{\hbar}{\Psi(\boldsymbol{r}, t)} \nabla \Psi(\boldsymbol{r}, t) \times \widehat{\boldsymbol{k}}=\frac{\hbar}{a_{0}} \sin \theta \hat{e}_{\phi}
$$

where $\hat{e}_{r}, \hat{e}_{\theta}$, and $\hat{e}_{\phi}$ are the radial, polar and azimuthal unit vectors and the electrons is in a spinup state. Because the momentum has no radial or polar momentum, the electron travels in circles of fixed radius around the nucleus, except at the stationary points located at $\theta=0, \pi$. Figure 1 illustrates this behavior.

\section{Equations of motion}

SD-QHD as formulated above and in Refs [57]-[60] works well if the electron's momentum and trajectory have closed form solutions. However, in order to push SD-QHD beyond atoms and use it to examine molecular orbitals (MOs) where such solutions do not exist, we have derived new equations of motion for SD-QHD which form the basis of a useful, numerical methodology. This is not trivial since the non-relativistic limit of the Dirac equation does not produce a spin-dependent force governing the time-dependence of the spin-dependent momentum. Therefore, we derive the equations-of-motion by constructing a free-particle Lagrangian $\left(F_{C l}+\right.$ $F_{Q}=0$ ) subject to the constraints placed upon the electron by its spin-dependent momentum, $\nabla \log \rho \times \mathbf{s}$. These constraints are that (a) the particle never leaves the level surface of $\rho$ and (b) the particle's momentum is always perpendicular to the spin vector, $\mathbf{s}$. The constraints are enforced using Lagrange multipliers:

$$
\mathcal{L}=\frac{1}{2} m \boldsymbol{v} \cdot \boldsymbol{v}+\Lambda\{\rho(\boldsymbol{r}(t=0))-\rho(\boldsymbol{r}(t))\}+\lambda\{z(t=0)-z(t)\}
$$


(Note, we placed the spin vector parallel to the z-axis.)

The subsequent Euler-Lagrange equations give

$$
\boldsymbol{F}=\Lambda \nabla \rho+\lambda \widehat{\boldsymbol{k}}=m \boldsymbol{a} .
$$

The multipliers $\Lambda$ and $\lambda$ can be solved by recalling that

$$
\frac{d z}{d t}=\frac{d^{2} z}{d t^{2}}=\frac{d \rho}{d t}=\frac{d^{2} \rho}{d t^{2}}=0
$$

The first two equalities in Eq. (18) combined with Eq. (17) give

$$
\lambda=-\Lambda \frac{\partial \rho}{\partial z} .
$$

Eq. (19)

$\lambda$ ensures that the velocity and the acceleration in the z-direction are zero. The second two equalities in Eq. (18) combined with Eq. (17) give:

$$
\Lambda=-m \frac{\boldsymbol{v}^{T} \widehat{H}_{\rho} \boldsymbol{v}}{\left(\frac{\partial \rho}{\partial x}\right)^{2}+\left(\frac{\partial \rho}{\partial y}\right)^{2}}
$$

where $\mathbf{v}$ is velocity and $\widehat{H}_{\rho}$ is the Hessian matrix of $\rho . \Lambda$ ensures that the particle stays on an isosurface of $\rho$. Therefore, the SD-QHD equations of motion are

$$
\boldsymbol{F}=-\left\{\frac{1}{m} \frac{\boldsymbol{p}^{T} \widehat{H}_{\rho} \boldsymbol{p}}{\left(\frac{\partial \rho}{\partial x}\right)^{2}+\left(\frac{\partial \rho^{2}}{\partial y}\right)}\right\}\left\{\frac{\partial \rho}{\partial x} \hat{\boldsymbol{\imath}}+\frac{\partial \rho}{\partial y} \hat{\boldsymbol{\jmath}}\right\}=m \boldsymbol{a},
$$

Eq. (21)

where $\mathbf{p}$ is the spin-dependent momentum. This force is unchanged if the Lagrange multiplier constrains the electron to the surface of $\log \rho$ or $\Psi$. We also note that the Lagrange multiplier in Eq. (20) is functionally similar to the curvature of the level curves of $\rho$, and the force in Eq. (21) points in the same direction as the curvature vector [64]. See the SI for more details.

Equation (21) presents a unique challenge for numerical integration techniques since (a), the equations of motion are not conservative (the force does not originate from the gradient of a potential energy) and (b), the force is velocity dependent. Thus, we use a modified version of the velocity-Verlet algorithm[65], [66]. We update the position with the standard expression, namely 


$$
\boldsymbol{r}_{i+1}=\boldsymbol{r}_{i}+\boldsymbol{v}_{i} \Delta t+\frac{1}{2 m} \boldsymbol{F}_{i} \Delta t^{2}
$$

The velocity is updated using Eq. (13),

$$
\mathbf{v}_{i+1}=\frac{1}{m} \nabla \log \rho\left(\mathbf{r}_{i+1}\right) \times \mathbf{s}
$$

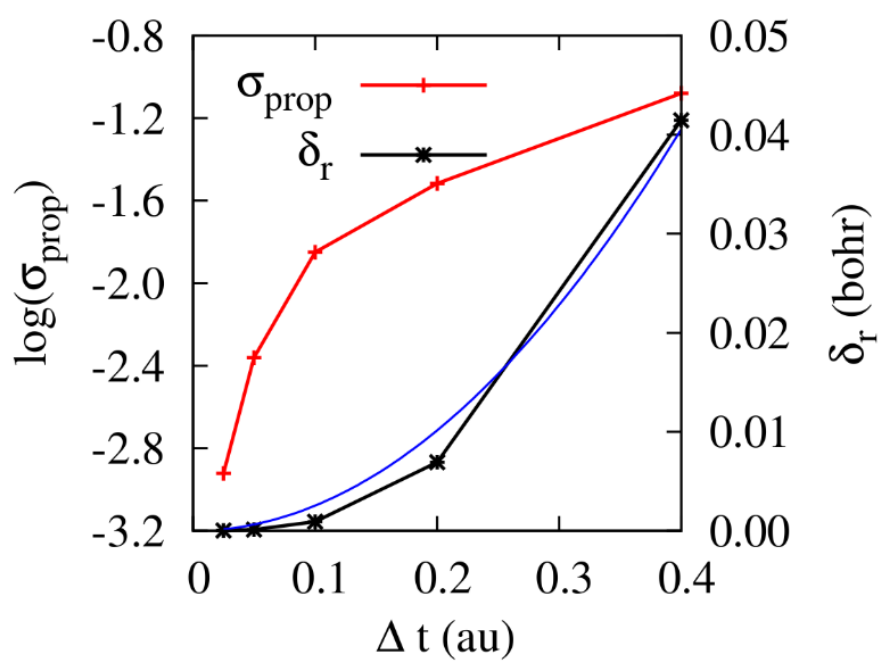

Figure 2 Error accumulated over one orbit using the numeric propagation scheme. The $\mathrm{x}$-axis is in atomic time units, the left y-axis is the logarithm (base 10) of the error calculated by Eq. (24) $\left(\sigma_{\text {prop }}\right)$ and the right y-axis is the fluctuations in bohr of the electron's orbital $\left(\delta_{r}\right)$. The blue line represents a quadratic function of the form $a \Delta t^{2}$. The electron starts $0.5 \mathrm{bohr}$ from the nucleus.

This algorithm is benchmarked against the analytical trajectories of the hydrogen atom [57].

The error was quantified from two perspectives. First, we examined the error accumulated over one orbit calculated by:

$$
\sigma_{\text {prop }}=\frac{1}{T} \int_{0}^{T}\left\|\boldsymbol{r}(t)_{\text {exact }}-\boldsymbol{r}(t)_{\text {approx }}\right\|_{2},
$$

where $\|\ldots\|_{2}$ is the $\mathrm{L}^{2}$ norm and $\mathrm{T}$ is the time for one orbit of the electron. Second, we measured the fluctuations of the orbital radius, $\delta_{\mathrm{r}}=\sqrt{\left\langle r^{2}\right\rangle-\langle r\rangle^{2}}$. The analytic solutions show that the orbital radius is constant. The electron was placed 0.5 bohr from the nucleus in in the $z=0$ plane. The quadratic dependence of the fluctuations of the electron's orbital radius with $\Delta t$ indicates that our scheme is a third order technique, like standard velocity-Verlet[67]. 

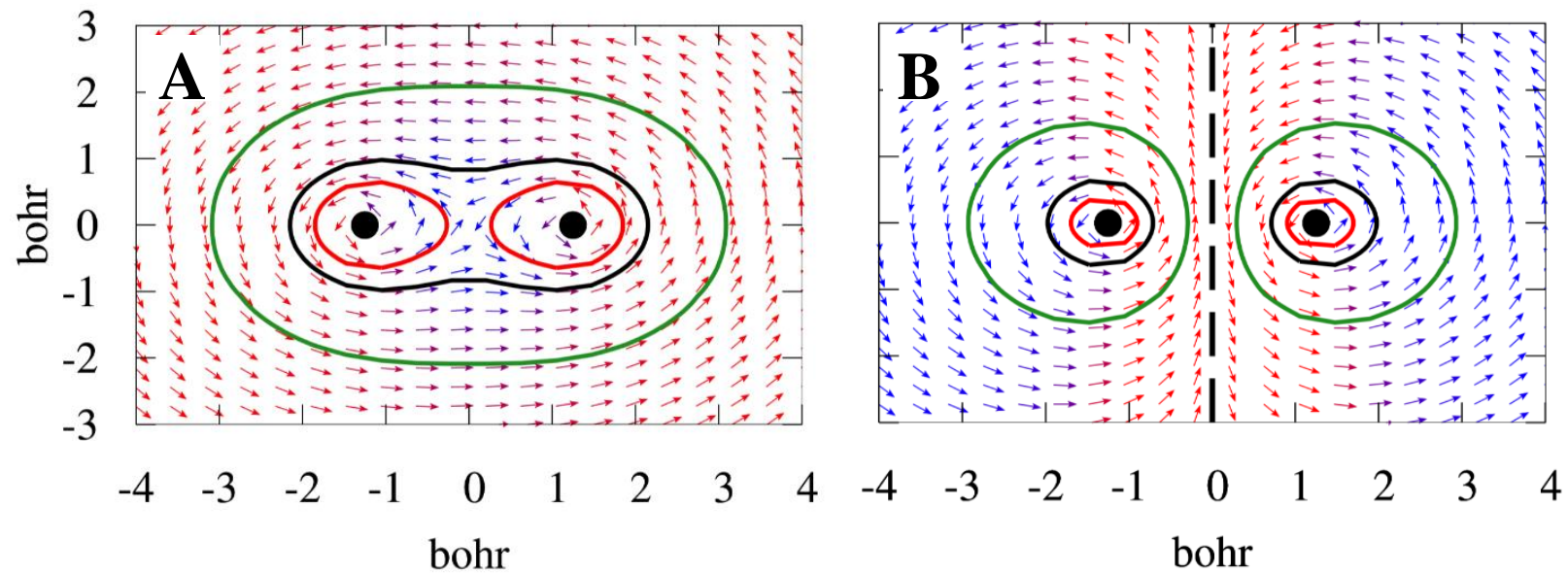

Figure 3 Contours of the $\mathrm{H}_{2}{ }^{+}$(A) bonding and (B) antibonding $\mathrm{MO}$ densities in the $z=0$ plane. The arrows represent $\nabla \log \rho \times s$, the momentum vector field of the electron. The momentum vectors are all scaled to the same length but are colored according to their magnitude. Red is larger, whereas blue is smaller. The thick, dotted line in B marks the wavefunction node. The nuclei are the black dots and are $2.5 \mathrm{bohr}$ apart, the energy minimum. The electron orbits along contours, none of which are circular.

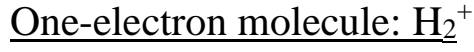

As a first application, we compute trajectories in the molecular orbitals for the one-electron molecule, $\mathrm{H}_{2}{ }^{+}-$a system commonly studied in undergraduate physical chemistry[68]. Approximate solutions are generated by implementing the Born-Oppenheimer approximation and by assuming that the MOs are a linear combination of $1 \mathrm{~s}$ orbitals centered on each nucleus, labeled $\mathrm{A}$ and $\mathrm{B}$ :

$$
\Psi_{ \pm}=\frac{1}{\sqrt{2}}\left(1 s_{A} \pm 1 s_{B}\right)
$$

The positive linear combination is the bonding $\mathrm{MO}$ and the negative linear combination is the antibonding MO. Eq. (25) is not an exact solution to the $\mathrm{H}_{2}{ }^{+}$Schrödinger equation[69]-[72] so $\nabla S \neq$ 0 . However, we use this simple approximation since its properties are similar to those of a more accurate wavefunction and since its spin-dependent momentum can be clearly derived. The derivation uses spherical coordinates $\left(\hat{e}_{r}, \hat{e}_{\theta}, \hat{e}_{\phi}\right)$ and places nucleus $\mathrm{A}$ at the origin. The molecular axis is parallel to the $x$-axis, the electron is spin-up, in the $z=0$ plane, and the spin vector is parallel to the $z$-axis:

$$
\boldsymbol{s}=-\frac{\hbar}{2} \hat{e}_{\theta}
$$

(see the SI for the definition of the coordinate system and a more detailed derivation). The spindependent momentum for the $\mathrm{H}_{2}{ }^{+}$electron is: 


$$
\boldsymbol{p}_{ \pm}=\nabla \log \rho \times \boldsymbol{s}=\frac{\hbar}{\Psi_{ \pm} a_{0} \sqrt{2}}\left\{\mp \frac{R \sin \phi}{r_{B}} 1 s_{B} \hat{e}_{r}+\left(1 s_{A} \pm \frac{r-R \cos \phi}{r_{B}} 1 s_{B}\right) \hat{e}_{\phi}\right\}
$$

Eq. (27)

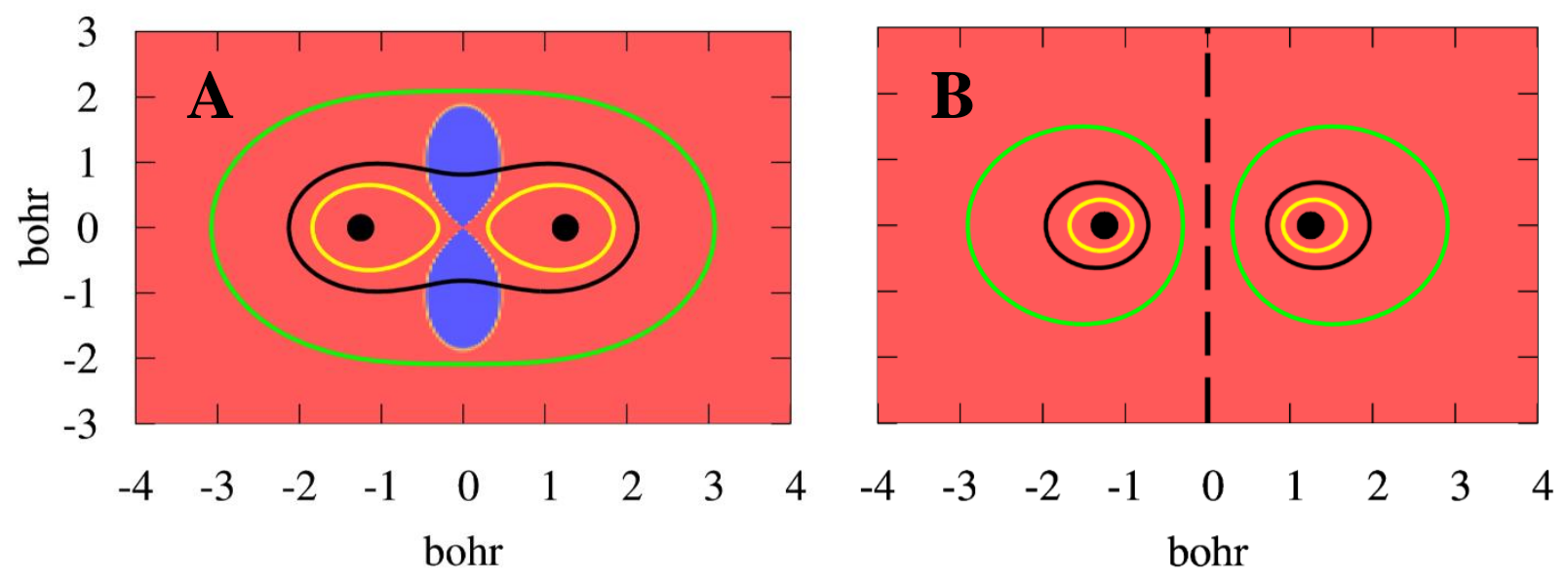

Figure 4 Contours of the $\mathrm{H}_{2}{ }^{+}(\mathrm{A})$ bonding and (B) antibonding $\mathrm{MO}$ densities in the $z=0$ plane overlaid with a colormap of the Lagrange multiplier, $\Lambda$. The thick, dotted line in $\mathrm{B}$ marks the wavefunction node. The nuclei are the black dots and are $2.5 \mathrm{bohr}$ apart. The colormap is blue in regions where $\Lambda$ is negative and is red where $\Lambda$ is positive. Only the bonding MO has negative values. These occur in regions of significant constructive overlap between the atomic orbitals on each atom.

where $R$ is the distance between the nuclei, $r_{B}$ is the distance from nucleus $\mathrm{B}$ to the electron and $a_{0}$ is the Bohr radius. Unlike the $1 s$ electron in the $\mathrm{H}$ atom, the $\mathrm{H}_{2}{ }^{+}$electron moves in the radial $\left(\hat{e}_{r}\right)$ direction. Therefore, the orbits are not circular, as shown in Fig. 3. The presence of the additional nucleus in $\mathrm{H}_{2}{ }^{+}$compared to the $\mathrm{H}$ atom perturbs the orbit and allows for the formation of a covalent bond. In the bonding molecular orbital, there are trajectories where the electron orbits both nuclei, conforming to the common analogy that covalent bonds share electrons. There are also trajectories that orbit single nuclei, or maxima near the nuclei. In the antibonding $\mathrm{MO}$, the electron never orbits both nuclei. It only orbits single maxima near each nucleus. (Although, the wavefunction maximum occurs at the nucleus in the $\mathrm{H}$ atom, the maxima shift slightly off the nucleus in the $\mathrm{H}_{2}{ }^{+}$molecule.) There is also a stationary point in the center between the nuclei for the bonding MO, where $r=r_{B}=\frac{R}{2}, \phi=0$, and $1 s_{A}=1 s_{B}$. This represents a saddle point in the MO. In the anti-bonding MO, there is a node bisecting the molecular axis which the electron cannot cross and where the momentum approaches infinity.

Next, we examine the Lagrange multiplier, $\Lambda$. As previously noted, $\Lambda\left\{\frac{\partial \rho}{\partial x} \hat{\boldsymbol{l}}+\frac{\partial \rho}{\partial y} \hat{\boldsymbol{j}}\right\}$ is the force required to keep the electron on the contour of $\rho$. So, when $\Lambda$ is positive, the force pushes "uphill" (increasing $\rho$ ) and, when $\Lambda$ is negative, the force pushes downhill (decreasing $\rho$ ). $\Lambda$ is also related to the curvature of the level curves of $\rho$. Therefore, if $\Lambda$ changes sign along a contour of $\rho$, it signifies a changing orientation of the curvature vector and circle tangent to the curve. In other words, if $\Lambda$ changes sign along a contour, the contour changes orientation. Figure 4 illustrates this observation. 
Figure 4 shows that $\Lambda$ is negative (blue) in regions of the bonding MO where the contours start to bend in. These indicate regions of significant, positive overlap between the atomic orbitals centered on the nuclei $\left(1 \mathrm{~s}_{\mathrm{A}}\right.$ and $\left.1 \mathrm{~s}_{B}\right)$. In this example, $\Lambda$ changes sign only along contours in regions where electron density is shared between the nuclei. Counter examples are shown in Fig. 5 and Figs. S2 and S3 in the SI. However, in all cases, $\Lambda$ changes sign along contours between nuclei only if electron density is shared. Therefore, $\Lambda$ doesn't change sign along contours in the MO in Fig 4B, since it has no constructive overlap or shared electron density. Finally, we highlight that the point in Fig. 4A between the nuclei where the two blue lobes meet, $\Lambda=0$ and is a saddle point. This occurs only in the bonding orbital, since the anti-bonding orbital in Fig $4 \mathrm{~B}$ has a node between the nuclei.

$\underline{\text { General Molecular Orbital Trajectories }}$
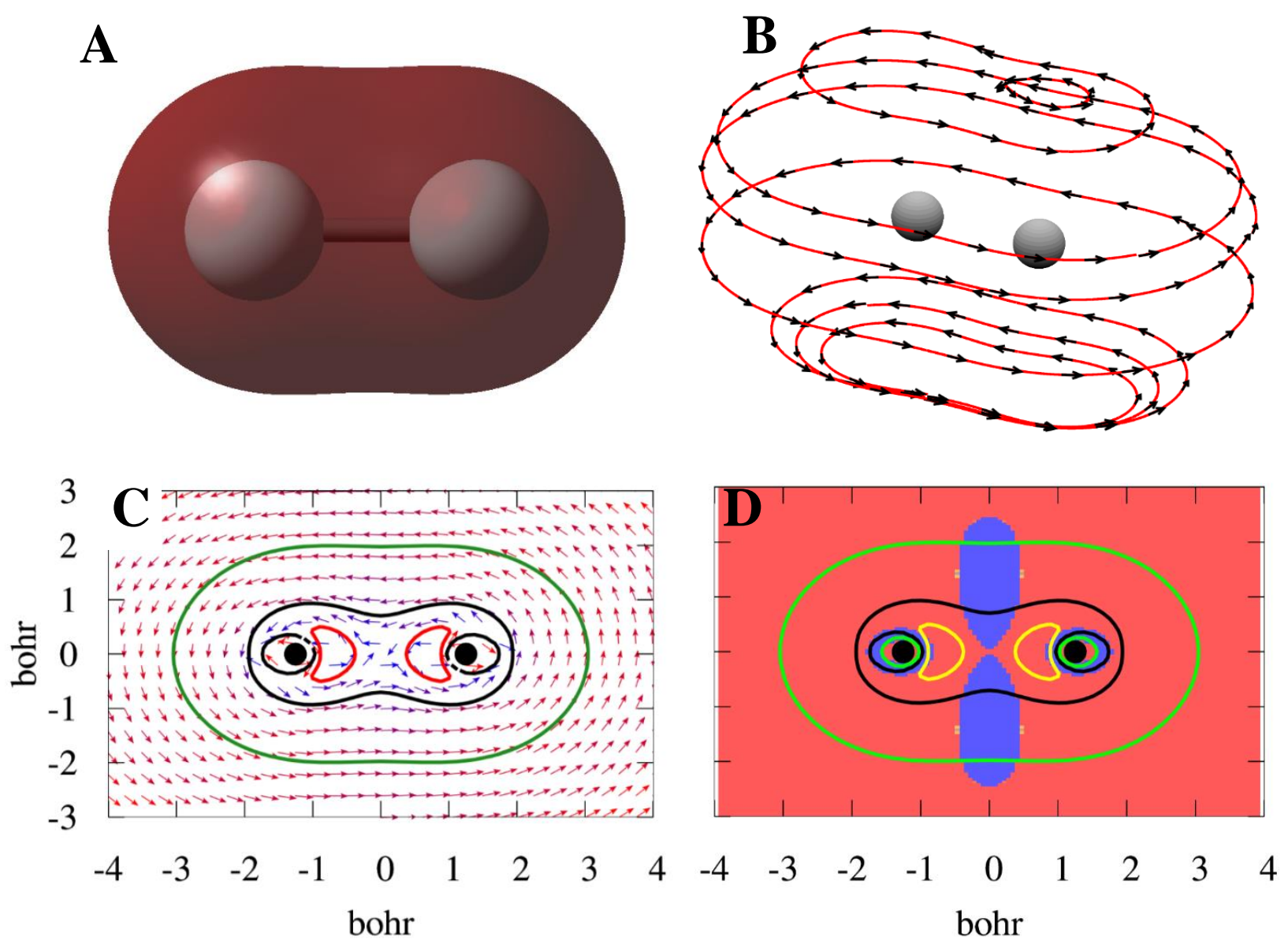

Figure 5 The $\sigma_{\mathrm{g}}$ molecular orbital of $\mathrm{F}_{2}$ shown as $(\mathrm{A})$ an isosurface, (B) randomly placed trajectories on the isosurface, $(\mathrm{C})$ the momentum field with $\rho$ contours in the $z=0$ plane, and (D) $\Lambda$ with $\rho$ contours in the $z=0$ plane. The spheres in $\mathrm{A}$ and $\mathrm{B}$ and the dots in $\mathrm{C}$ and $\mathrm{D}$ represent the nuclei. The arrows in $\mathrm{B}$ and $\mathrm{C}$ represent the momentum vectors, which are normalized and scaled. In C, they are colored according to their magnitude (blue is slower and red is fast). The colormap in D blue in regions where $\Lambda$ is negative and is red where $\Lambda$ is positive. The radial nodes are close to the nucleus and are covered by the dots in $\mathrm{C}$ and D. 
As a first step towards a general method of electron trajectories in molecular orbitals of multi-electron systems, we start with the Hartree-Fock (HF) approximation. HF theory is a useful starting point since the eigenstates of the HF Hamiltonian are canonical MOs [73]. In other words, the canonical MOs can be used to construct any solution to the TDSE in which the HF Hamiltonian is appropriate. Therefore, we use the equations-of-motion in Eq. (21) and the numerical integration scheme in Eqs. (22) and (23) to generate electron trajectories for eigenfunctions of the HF Hamiltonian. Finally, we note that this formalism works for Kohn-Sham DFT and semiempirical methods, since their MOs are the eigenfunctions of the Kohn-Sham and semiempirical Hamiltonian.

\section{$\underline{F_{2}}$ and Benzene}

As illustrative calculations, we examine the trajectories of electrons in the MOs of molecular fluorine and benzene. The $\mathrm{F}_{2}$ MOs are generated using the HF/aug-cc-pvqz level of theory and the benzene MOs are calculated using HF/cc-pvtz. Both are calculated with the
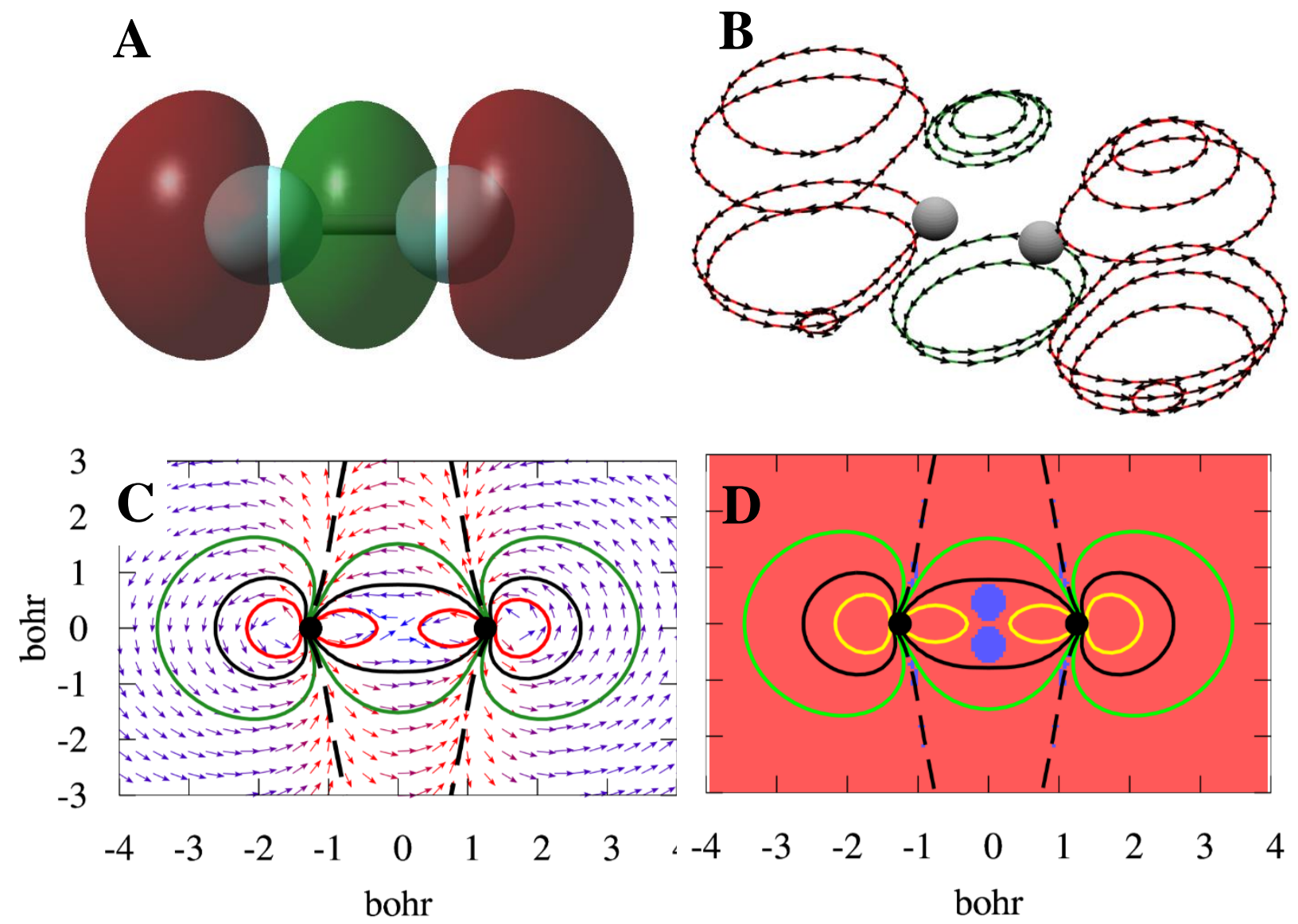

Figure 6 The $2 \sigma_{\mathrm{g}}$ molecular orbital of $\mathrm{F}_{2}$ shown as (A) an isosurface, (B) randomly placed trajectories on the isosurface, (C) the momentum field with $\rho$ contours in the $z=0$ plane, and (D) $\Lambda$ with $\rho$ contours in the $z=0$ plane. The spheres in $\mathrm{A}$ and $\mathrm{B}$ and the dots in $\mathrm{C}$ and $\mathrm{D}$ represent the nuclei. The arrows in $\mathrm{B}$ and $\mathrm{C}$ represent the momentum vectors, which are normalized and scaled. In $\mathrm{C}$, they are colored according to their magnitude (blue is slower and red is fast). The dotted lines in C and D indicate the node. The colormap in $\mathrm{D}$ is blue in regions where $\Lambda$ is negative and is red where $\Lambda$ is positive. 
Gaussian09 suite of programs[74] and the wavefunction is printed out in the AIMAll WFX format[75] after the molecular geometry is optimized. In each case, we assume the wavefunction is exact and the electron only has spin-dependent momentum. This approximation improves as the basis set size increases. The trajectories are then generated using our freely-available, SD-QHD code[76]. Input files for this code are included in the SI. In the case of fluorine, the molecular axis is parallel to the $\mathrm{x}$-axis and the benzene is in the $z=0$ plane. The spin vector is parallel to the $\mathrm{z}$ axis and only $\alpha$ electrons are considered. We used the code to generate contours of the MO probability density, the spin-dependent momentum field, trajectories randomly placed on an isosurface, and color maps of $\Lambda$. The MO contours, momentum fields, and $\Lambda$ colormaps
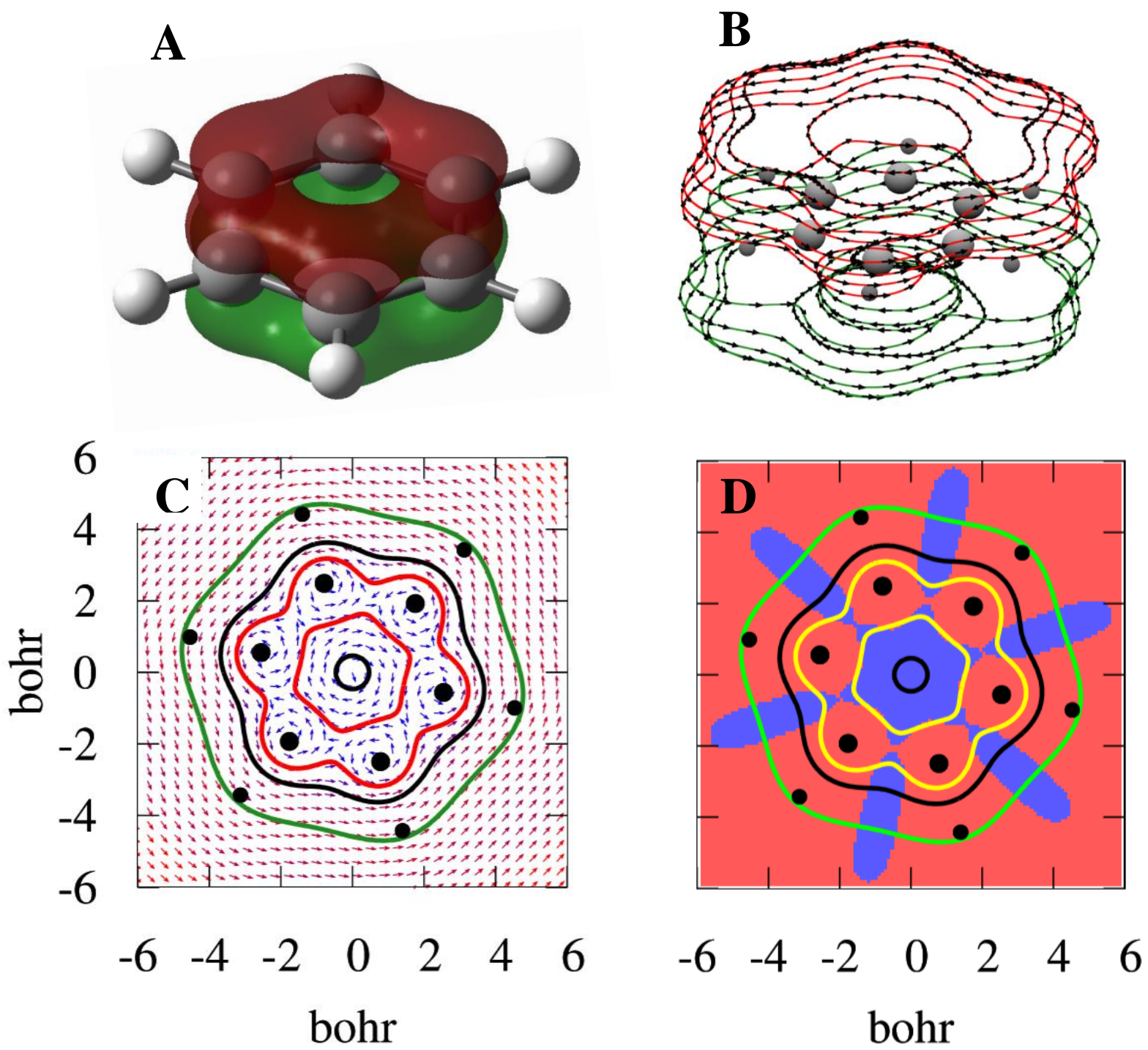

Figure 7 The $\mathrm{a}_{2 \mathrm{u}}$ molecular orbital of benzene shown as (A) an isosurface, (B) randomly placed trajectories on the isosurface, (C) the momentum field with $\rho$ contours in the $z=1$ plane, and (D) $\Lambda$ and $\rho$ contours in the $z=1$ plane. The spheres in A and B and the dots in $\mathrm{C}$ and $\mathrm{D}$ represent the nuclei. The arrows in $\mathrm{B}$ and $\mathrm{C}$ represent the momentum vectors, which are normalized and scaled. In $\mathrm{C}$, they are colored according to their magnitude (blue is slower and red is fast). The colormap in D is blue in regions where $\Lambda$ is negative and is red where $\Lambda$ is positive. 
calculations were performed on $200 \times 200 \times 100$ Cartesian grids that extended 5 bohr beyond the nuclei.

Figures 5 through 8 illustrate how the standard picture of molecular orbitals changes when represented using SD-QHD. In Figs. 5 and 6, the $\sigma_{g}$ and $2 \sigma_{g}$ MOs of the $\mathrm{F}_{2}$ molecule are examined. Both are bonding MOs and display behavior like what may be predicted from the model $\mathrm{H}_{2}{ }^{+}$ calculation. Specifically, the momentum field in Fig. 5C shows some electron trajectories orbiting both nuclei and some orbiting one local maximum near one of the nuclei. Since $\sigma_{\mathrm{g}}$ has radial nodes there are also some orbits around local maxima between the nuclei (see red contour in Fig 5C).
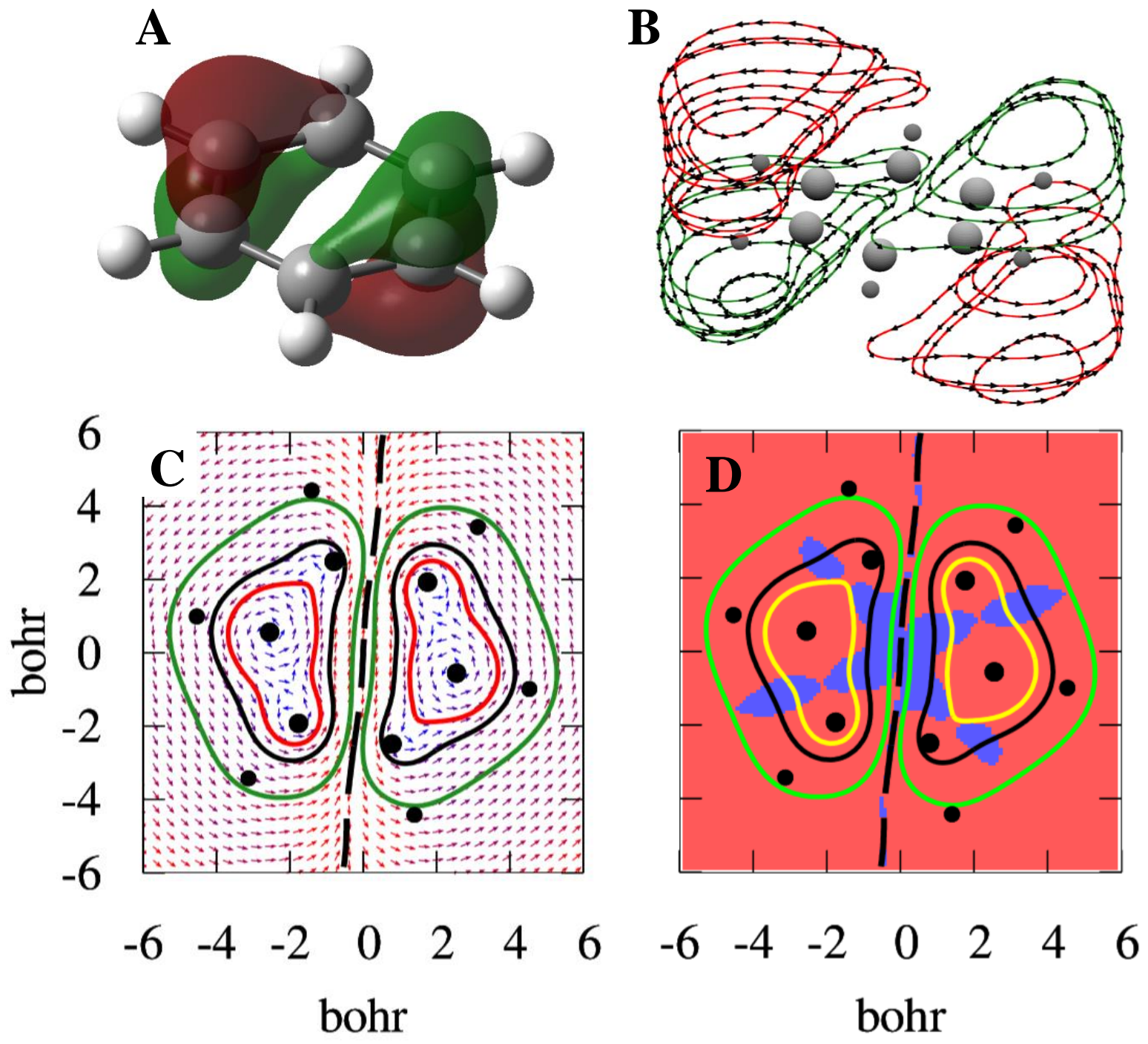

Figure 8 One of the degenerate $e_{1 g}$ molecular orbitals of benzene shown as (A) an isosurface, (B) randomly placed trajectories on the isosurface,(C) the momentum field with $\rho$ contours in the $z=1$ plane, and (D) $\Lambda$ with $\rho$ contours in the $z=1$ plane. The spheres in $\mathrm{A}$ and $\mathrm{B}$ and the dots in $\mathrm{C}$ and $\mathrm{D}$ represent the nuclei. The arrows in $\mathrm{B}$ and $\mathrm{C}$ represent the momentum vectors, which are normalized and scaled. In $\mathrm{C}$, they are colored according to their magnitude (blue is slower and red is fast). The dotted lines in $\mathrm{C}$ and $\mathrm{D}$ indicate the node. The colormap in $\mathrm{D}$ is blue in regions where $\Lambda$ is negative and is red where $\Lambda$ is positive. 
The momentum field in the $2 \sigma_{\mathrm{g}}$ orbital in Fig. $6 \mathrm{C}$ shows slightly different behavior since there are nodes near the nuclear positions, preventing trajectories from orbiting any nuclei. Here, the trajectories swirl around four maxima, none of which are centered on the nuclei. Two maxima are on either side of the nuclei and two maxima are between the nuclei.

$\Lambda$ behaves similarly in both Figs. 5D and 6D. Specifically, both MOs have regions of positive overlap between atomic orbitals centered on each nucleus. Therefore, $\Lambda$ changes sign along MO contours and the sign change occurs between the nuclei. $\Lambda$ also changes sign along contours close to the nuclei. This is due to the presence of the nodes. Finally, $\Lambda$ has a stationary point between the nuclei that occurs at a saddle point in the MO density in both the $\sigma_{g}$ and $2 \sigma_{g}$ MOs.

Figures 7 and 8 show the $\mathrm{a}_{2 u}$ and $\mathrm{e}_{1 \mathrm{~g}} \pi$-bonding MOs in benzene using the SD-QHD formalism. These MOs are both bonding both have trajectories that orbit multiple nuclei. In Fig. 7C, trajectories orbit maxima near each of the carbon nuclei, whereas in Fig. 8C, some carbon nuclei are not orbited. This is due to the presence of the node bisecting the benzene ring. Finally, there are trajectories in Fig. 7C that orbit the local minimum in the center of the ring. Since these are bonding MOs, Figs. 7D and 8D show that $\Lambda$ changes sign along contours between the carbon atoms. Again, this occurs because wavefunction density is shared between the nuclei. $\Lambda$ also has a saddle point between nuclei that share electron density. Interestingly, the saddle points only occur between the carbon atoms, since it is the carbon $p_{z}$ orbitals that form the bonds.

\section{Conclusions}

Trajectory methods have proven to be useful tools in chemical physics. However, they are rarely applied to multi-electron, stationary state, molecular wavefunctions. Here, we have built upon a spin-dependent formulation of quantum hydrodynamics (SD-QHD)[57]-[60] and developed a method that calculates electron trajectories in molecular orbitals. These calculations rely on a numerical integration scheme that uses equations of motion based on constraints enforced by a Lagrange multiplier, $\Lambda$. The trajectories and $\Lambda$ have may interesting features that warrant further examination.

Specifically, trajectories in bonding MOs share the property that they all have stationary points between bonded nuclei. At these points, the electron is motionless because the MO density has a local maximum or a saddle point. Local maxima occur if the overlap between the atomic orbitals is strong. For example, if two p-atomic orbitals form a $\sigma$-bond and overlap such that the maxima of their lobes coincide, the corresponding MO density will have a local maximum, instead of a saddle point (see Fig S3 in the SI). Saddle points occur when the MO density retains the distinct maxima of the corresponding atomic orbitals. The presence of these stationary points between nuclei is reminiscent of the atoms-in-molecules (AIM) approach that assigns bonds in an electron-density map by connecting local maxima in the total electron density (atoms) to saddle points, which typically occur between atoms[77], [78].

The Lagrange multiplier, $\Lambda$, also has intriguing properties. When $\Lambda$ changes sign along a contour, it signals that the contour changes orientation, which occurs between atoms only if MO density is shared between two nuclei. (There is an exception if there is very strong overlap.) 
Interestingly, if the MO density has a saddle point between two nuclei, then $\Lambda$ also has a saddle point. Furthermore, $\Lambda$ equals zero at these points, meaning the MO contour has no curvature and there is no force on the electron. Again, the location of this unique point in $\Lambda$ echoes the atoms-inmolecules approach to identifying covalent bonds[77], [78].

Finally, SD-QHD offers a different perspective on the description of electrons in molecules. Instead of an electron cloud, the SD-QHD pictures imagines electrons traveling along real trajectories in a "fluid" governed by the quantum continuity equation. In bonding MOs, the electron travels along paths formed by combining atomic orbitals. The effect is that the new paths are "shared" by the atoms. In anti-bonding MOs, the electron trajectories are not shared between atoms due to the presence of MO nodes, which the electrons cannot cross. Based on these initial observations, further studies of electron trajectories may provide unique insights into the nature of the chemical bond.

\section{Author Contributions}

IS conceived and supervised the study and designed the experiments. IS and HA conducted the experiments. IS wrote the manuscript.

\section{Acknowledgements}

This work was supported by an NSF-REU CHE-1062629; The MERCURY consortium (http://mercuryconsortium.org/) under NSF grants CHE-1229354 and CHE-1662030.

\section{Supporting Information}

The relationship between $\Lambda$ and curvature; a derivation of a simplified force expression; a more detailed derivation of the $\mathrm{H}_{2}{ }^{+}$electron momentum; additional trajectories in excited state $\mathrm{H}_{2}{ }^{+}$ molecular orbitals; and input files for the SD-QHD code are found in the SI. 


\section{References}

[1] L. de Broglie, C. R. Acad. Sci., 1926, 183, 447.

[2] E. Madelung, Zeitschrift für Phys., 1927, 40, 322-326.

[3] D. Bohm, Phys. Rev., 1952, 85, 166-179.

[4] D. Bohm, Phys. Rev., 1952, 85, 180-183.

[5] P. R. Holland, in The Quantum Theory of Motion: An Account of the de Broglie-Bohm Causal Interpretation of Quantum Mechanics; Cambridge University Press, , 1995.

[6] C. J. Trahan, R. E. Wyatt, in Quantum Dynamics with Trajectories: Introduction to Quantum Hydrodynamics; Springer New York, , 2005.

[7] D. F. Styer, M. S. Balkin, K. M. Becker, M. R. Burns, C. E. Dudley, S. T. Forth, J. S. Gaumer, M. A. Kramer, D. C. Oertel, L. H. Park, M. T. Rinkoski, C. T. Smith, T. D. Wotherspoon, Am. J. Phys., 2002, 70, 288-297.

[8] E. Gindensperger, C. Meier, J. A. Beswick, J. Chem. Phys., 2000, 113, 9369-9372.

[9] O. V Prezhdo, C. Brooksby, Phys. Rev. Lett., 2001, 86, 3215-3219.

[10] I. Burghardt, K. B. Møller, G. Parlant, L. S. Cederbaum, E. R. Bittner, Int. J. Quantum Chem., 2004, 100, 1153-1162.

[11] S. Garashchuk, M. V Volkov, Mol. Phys., 2012, 110, 985-993.

[12] S. Garashchuk, J. Jakowski, L. Wang, B. G. Sumpter, J. Chem. Theory Comput., 2013, 9, 5221-5235.

[13] S. Garashchuk, J. Jakowski, V. A. Rassolov, Mol. Simul., 2015, 41, 86-106.

[14] R. E. Wyatt, Chem. Phys. Lett., 1999, 313, 189-197.

[15] R. E. Wyatt, J. Chem. Phys., 1999, 111, 4406-4413.

[16] S. W. Derrickson, E. R. Bittner, B. K. Kendrick, J. Chem. Phys., 2005, 123, 54107.

[17] C.-C. Chou, R. E. Wyatt, Phys. Rev. E, 2006, 74, 66702.

[18] C.-C. Chou, R. E. Wyatt, J. Chem. Phys., 2008, 128, 154106.

[19] C.-C. Chou, Chem. Phys., 2015, 457, 160-170.

[20] C.-C. Chou, Int. J. Quantum Chem., 2015, 115, 419-425.

[21] C.-C. Chou, Ann. Phys. (N. Y)., 2018, 393, 167-183.

[22] Y. Wang, C.-C. Chou, Chem. Phys. Lett., 2018, 699, 125-131.

[23] N. Zamstein, D. J. Tannor, J. Chem. Phys., 2012, 137, 22 A517.

[24] N. Zamstein, D. J. Tannor, J. Chem. Phys., 2012, 137, 22A518.

[25] S. Garashchuk, Chem. Phys. Lett., 2010, 491, 96-101. 
[26] S. Garashchuk, V. A. Rassolov, J. Chem. Phys., 2003, 118, 2482-2490.

[27] R. E. Wyatt, C. L. Lopreore, G. Parlant, J. Chem. Phys., 2001, 114, 5113-5116.

[28] J. C. Burant, J. C. Tully, J. Chem. Phys., 2000, 112, 6097-6103.

[29] E. R. Bittner, J. Chem. Phys., 2000, 112, 9703-9710.

[30] A. Benseny, G. Albareda, Á. S. Sanz, J. Mompart, X. Oriols, Eur. Phys. J. D, 2014, 68, 286.

[31] Y. Couder, S. Protière, E. Fort, A. Boudaoud, Nature, 2005, 437, 208.

[32] Y. Couder, E. Fort, Phys. Rev. Lett., 2006, 97, 154101.

[33] A. Eddi, J. Moukhtar, S. Perrard, E. Fort, Y. Couder, Phys. Rev. Lett., 2012, 108, 264503.

[34] D. M. Harris, J. Moukhtar, E. Fort, Y. Couder, J. W. M. Bush, Phys. Rev. E, 2013, 88, 11001 .

[35] J. Moláček, J. W. M. Bush, J. Fluid Mech., 2013, 727, 612-647.

[36] J. W. M. Bush, Annu. Rev. Fluid Mech., 2015, 47, 269-292.

[37] A. U. Oza, R. R. Rosales, J. W. M. Bush, Chaos An Interdiscip. J. Nonlinear Sci., 2018, $28,96106$.

[38] A. Andersen, J. Madsen, C. Reichelt, S. Rosenlund Ahl, B. Lautrup, C. Ellegaard, M. T. Levinsen, T. Bohr, Phys. Rev. E, 2015, 92, 13006.

[39] C. Levit, J. Sarfatti, 1998, G. Hunter, S. Jeffers, and J.-P. Vigier, Eds.; Springer Netherlands, Dordrecht, 1998.

[40] M. Kohout, Int. J. Quantum Chem., 2002, 87, 12-14.

[41] L. D. Site, Europhys. Lett., 2002, 57, 20-24.

[42] S. S. Iyengar, H. B. Schlegel, G. A. Voth, J. Phys. Chem. A, 2003, 107, 7269-7277.

[43] A. S. Sanz, X. Gimenez, J. M. Bofill, S. Miret-Artes, Time-Dependent Density Functional Theory from a Bohmian Perspective, Chemical Reactivity Theory: A Density Functional View. 2009.

[44] X. Oriols, Phys. Rev. Lett., 2007, 98, 66803.

[45] R. A. Leacock, M. J. Padgett, Phys. Rev. D, 1983, 28, 2491-2502.

[46] R. S. Bhalla, A. K. Kapoor, P. K. Panigrahi, Am. J. Phys., 1997, 65, 1187-1194.

[47] B. Poirier, D. Tannor, Mol. Phys., 2012, 110, 897-908.

[48] C.-D. Yang, Ann. Phys. (N. Y)., 2005, 319, 444-470.

[49] C.-D. Yang, Ann. Phys. (N. Y)., 2005, 319, 399-443.

[50] Y. Goldfarb, I. Degani, D. J. Tannor, J. Chem. Phys., 2006, 125, 231103. 
[51] C.-D. Yang, Ann. Phys. (N. Y)., 2006, 321, 2876-2926.

[52] C.-C. Chou, R. E. Wyatt, Phys. Rev. A, 2008, 78, 44101.

[53] C.-C. Chou, R. E. Wyatt, J. Chem. Phys., 2008, 128, 234106.

[54] C.-C. Chou, R. E. Wyatt, J. Chem. Phys., 2008, 129, 124113.

[55] B. Poirier, Phys. Rev. A, 2008, 77, 22114.

[56] C.-C. Chou, Chem. Phys. Lett., 2014, 591, 203-206.

[57] C. Colijn, E. R. Vrscay, Phys. Lett. A, 2002, 300, 334-340.

[58] C. Colijn, E. R. Vrscay, Found. Phys. Lett., 2003, 16, 303-323.

[59] C. Colijn, E. R. Vrscay, Phys. Lett. A, 2004, 327, 113-122.

[60] J. A. Timko, E. R. Vrscay, Found. Phys., 2009, 39, 1055-1071.

[61] R. Gurtler, D. Hestenes, J. Math. Phys., 1975, 16, 573-584.

[62] M. Nowakowski, Am. J. Phys., 1999, 67, 916-919.

[63] P. Strange, in Relativistic Quantum Mechanics: With Applications in Condensed Matter and Atomic Physics; Cambridge University Press, Cambridge, 1998.

[64] R. Goldman, Comput. Aided Geom. Des., 2005, 22, 632-658.

[65] L. Verlet, Phys. Rev., 1967, 159, 98-103.

[66] H. Gould, J. Tobochnik, C. Wolfgang, in An Introduction to Computer Simulation Methods: Applications to Physical Systems (3rd Edition); Addison-Wesley Longman Publishing Co., Inc., USA, 2005.

[67] M. P. Allen, M. P. Allen, D. J. Tildesley, T. ALLEN, D. J. Tildesley, in Computer Simulation of Liquids; Clarendon Press, , 1989.

[68] D. A. (Donald A. McQuarrie, in Physical chemistry: a molecular approach; Sausalito, Calif. : University Science Books, [1997] (C1997, , .

[69] L. Pauling, Chem. Rev., 1928, 5, 173-213.

[70] H. O. Pritchard, H. A. Skinner, J. Chem. Soc., 1951, 945-952.

[71] P. C. McKinney, J. Chem. Phys., 1965, 42, 3537-3539.

[72] C.-D. Yang, H.-J. Weng, Int. J. Quantum Chem., 2011, 111, 2980-2999.

[73] A. Szabo, N. S. Ostlund, in Modern Quantum Chemistry: Introduction to Advanced Electronic Structure Theory; Dover Publications, , 1996.

[74] G. E. S. M. J. Frisch, G. W. Trucks, H. B. Schlegel, V. B. M. A. Robb, J. R. Cheeseman, G. Scalmani, M. C. B. Mennucci, G. A. Petersson, H. Nakatsuji, G. Z. X. Li, H. P. Hratchian, A. F. Izmaylov, J. Bloino, K. T. G. Zheng, J. L. Sonnenberg, M. Hada, M. Ehara, Y. H. R. Fukuda, J. Hasegawa, M. Ishida, T. Nakajima, J. A. M. J. O. Kitao, H. 
Nakai, T. Vreven, E. B. J. E. Peralta, F. Ogliaro, M. Bearpark, J. J. Heyd, J. N. K. N. Kudin, V. N. Staroverov, R. Kobayashi, S. S. I. K. Raghavachari, A. Rendell, J. C. Burant, J. J. J. Tomasi, M. Cossi, N. Rega, J. M. Millam, M. Klene, J. E. Knox, J. B. Cross, V. Bakken, C. Adamo, A. J. A. R. Gomperts, R. E. Stratmann, O. Yazyev, R. L. M. R. Cammi, C. Pomelli, J. W. Ochterski, P. S. K. Morokuma, V. G. Zakrzewski, G. A. Voth, Ö. F. J. J. Dannenberg, S. Dapprich, A. D. Daniels, J. C. and D. J. F. J. B. Foresman, J. V. Ortiz, Gaussian 09, Revision E.01, Gaussian 09, Revision B.01, Gaussian, Inc., Wallingford CT. 2009.

[75] T. A. Keith, AIMAll (Version 10.10.12). http://aim.tkgristmill.com/.

[76] H. Anthony, I. Sumner, C++ code to compute electron trajectories in molecular orbitals using spin-dependent quantum hydrodynamics. DOI:10.5281/zenodo.3690174, 2020.

[77] R. F. W. Bader, in Atoms in Molecules: A Quantum Theory; Clarendon Press, , 1994.

[78] R. F. W. Bader, Acc. Chem. Res., 1985, 18, 9-15. 